\title{
Tuning viscoelastic properties of supramolecular peptide gels via dynamic covalent crosslinking $\dagger$
}

Cite this: Org. Biomol. Chem., 2015, 13, 1983

Received 18th October 2014,

Mohammad Aref Khalily, Melis Goktas and Mustafa O. Guler*

Accepted 19th December 2014

DOI: $10.1039 / c 4 o b 02217 c$

www.rsc.org/obc

A dynamic covalent crosslinking approach is used to crosslink supramolecular peptide gels. This novel approach facilitates tuning viscoelastic properties of the gel and enhances mechanical stability (storage modulus exceeding $10^{5} \mathrm{~Pa}$ ) of the peptide gels.

Supramolecular peptide gels are a class of supramolecular polymers, which are capable of encapsulating a large amount of water, up to 100 times of their dry weight. These soft materials are produced by molecular self assembly of naturally occurring short peptide sequences. ${ }^{1}$ Beside their inherent biocompatibility, biodegradability and bio-functionality peptides can be straightforwardly designed with a desired sequence, function and molecular weight. ${ }^{2}$ In soft materials research, versatile peptide gels have attracted a large amount of attention and they have been explored comprehensively. ${ }^{3}$ Supramolecular peptide gels have been exploited as scaffolds for wound healing, sustained release of drugs and biomolecules, cell culture media and tissue engineering, ${ }^{3 b, c, 4}$ templates for nanofabrication, ${ }^{5}$ catalysts for organic reactions, ${ }^{6}$ and light harvesting devices. ${ }^{7}$ Despite the versatility of peptide hydrogels, they suffer from low mechanical stability with storage moduli of

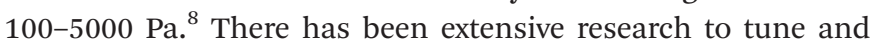
enhance the mechanical stability of peptide gels. Light, ${ }^{8}$ metal ions, ${ }^{9}$ disulfide, ${ }^{10}$ catalytic, ${ }^{11}$ and enzymatic ${ }^{12}$ crosslinking mechanisms are main approaches to improve the mechanical stability of peptide hydrogels.

Here, we have utilized a novel dynamic imine covalent bonding approach to crosslink the supramolecular peptide hydrogels to tune and enhance their viscoelastic characteristics. This approach is quite simple and straightforward. The crosslinking takes place between amine and aldehyde groups in aqueous medium at room temperature and forming a dynamic covalent bond, imine. ${ }^{13}$

Institute of Materials Science and Nanotechnology, National Nanotechnology Research Center (UNAM), Bilkent University, Ankara, Turkey 06800. E-mail: moguler@unam.bilkent.edu.tr; Fax: +90 312266 4365; Tel: +903122903552

$\dagger$ Electronic supplementary information (ESI) available: Experimental procedures and additional details are provided in the ESI. See DOI: 10.1039/c4ob02217c
This novel crosslinking system has many advantages over other crosslinking strategies; it does not require any catalyst, enzyme, photocrosslinker, metal ions and any other crosslinking agent. Moreover, there is no need for purification of crosslinked peptide gels since water is the only side product from the reaction of amine and aldehyde. The reversibility of the imine bond is crucial for suitable self-assembly of peptide molecules to conserve the nanofibrous morphology and selfhealing properties.

In this study, we have designed and synthesized a peptide amphiphile (PA) molecule (Lauryl-VVAGKK-Am, $\mathrm{K}_{2}$-PA) (Fig. 1A). At $\mathrm{pH}>7$, the PA molecules self-assemble into nanofibers with a diameter of $c a .10 \mathrm{~nm}$ (Fig. 5B, and $55 \dagger$ ) directed
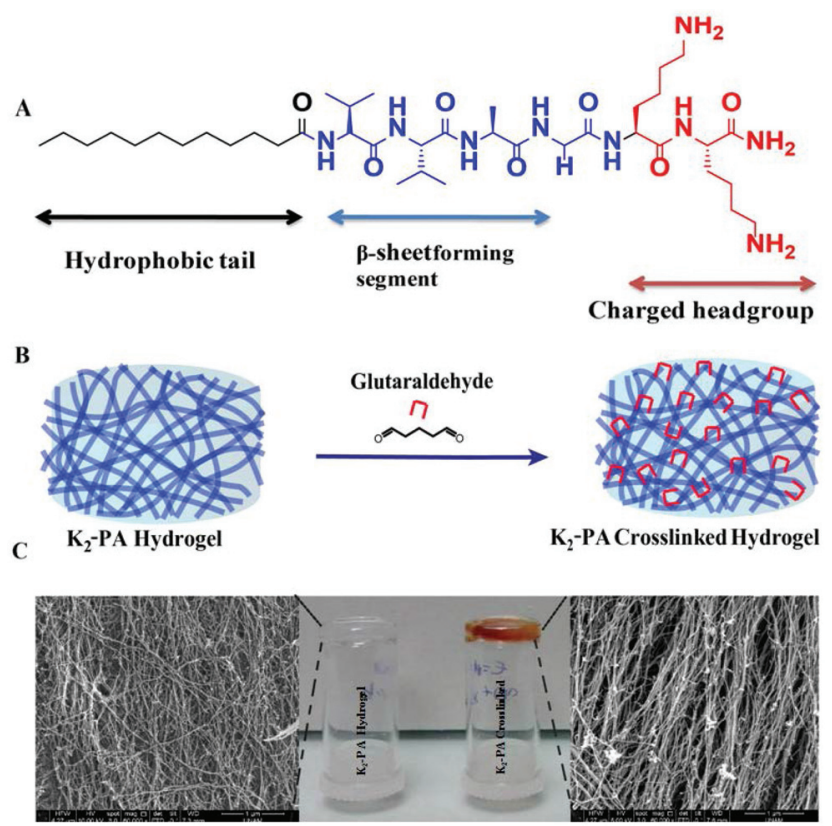

Fig. 1 Schematic representation of the peptide amphiphile chemical structure (A), peptide gel formation and a glutaraldehyde crosslinking strategy (B), peptide gel (left) and crosslinked peptide gel (right) (C). 
by $\beta$-sheet structures. A three-dimensional network of the PA nanofibers forms a self-supporting gel at concentrations of $1 \mathrm{wt} \%$ (Fig. 1C and S3a $\dagger$ ) or even lower (0.1 wt\%. Fig. S11 $\dagger$ ).

In this design, the lauryl group promotes hydrophobic interactions. Meanwhile, $\beta$-sheet forming amino acids (VVA) facilitate hydrogen-bonding. These two segments are required for self-assembly of peptide molecules in aqueous medium ${ }^{4}$ (Fig. 1C). Two lysine residues have significant roles in assisting solubility of the peptide molecules in water and then reacting with glutaraldehyde molecules to crosslink the peptide nanofibers (Fig. 1B).

We investigated the formation of the imine bond and its effect on peptide self-assembly and the $\beta$-sheet structure using UV-Vis and circular dichroism (CD) spectroscopy. Although glutaraldehyde is widely used for crosslinking of biomaterials, its chemistry is still not well understood. ${ }^{14}$ The reaction between amines and glutaraldehyde in aqueous solution is quite complex and many studies have been conducted to determine the products. ${ }^{14}$ After peptide gel formation at $\mathrm{pH} 7.5$, $10 \mu \mathrm{L}$ of glutaraldehyde (25\% solution) was added and then imine formation was followed by UV-Vis spectroscopy. Interestingly, the crosslinked supramolecular peptide gels showed absorbance at $265 \mathrm{~nm}$ (Fig. 2A), which proves the presence of the imine bond. ${ }^{15}$

We further investigated the effect of the crosslinking on peptide self-assembly by circular dichroism (CD). Dilute solution of peptide gels showed the $\beta$-sheet structure (a positive signal at $195 \mathrm{~nm}$ and a negative signal at $220 \mathrm{~nm}$ ) and glutaraldehyde crosslinking did not affect the $\beta$-sheet structure (Fig. 2B). This could be due to the nature of dynamic and reversible imine bond formation in aqueous media, which

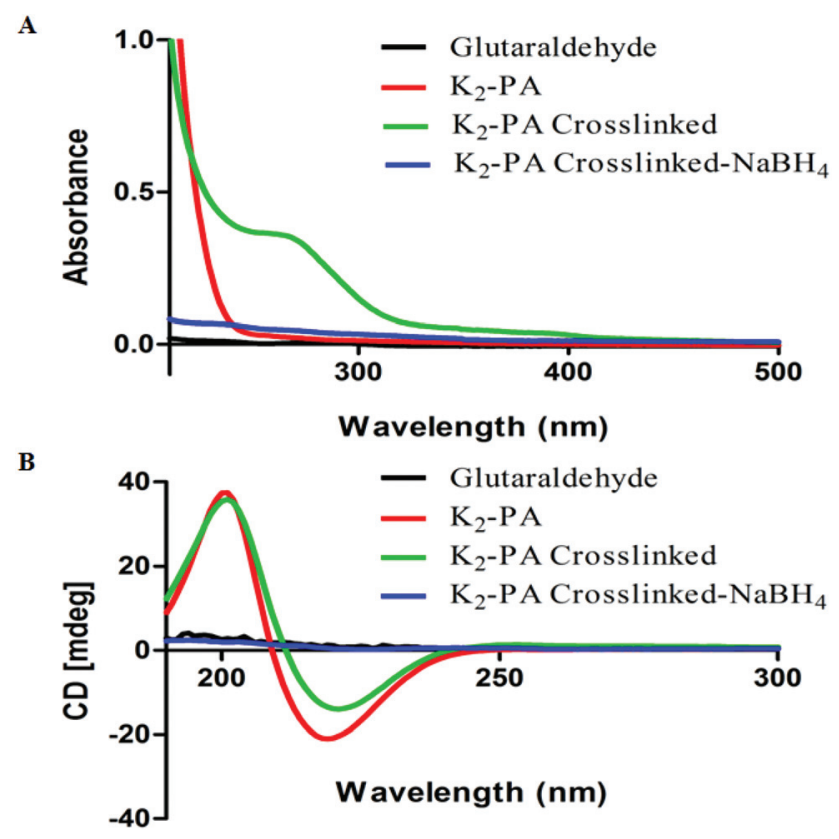

Fig. 2 UV-Vis spectroscopy (A) and circular dichroism (B) analysis of peptide gels and crosslinked peptide gels at $\mathrm{pH} 7.5$. does not disturb the peptide self-assembly. The imine can be reduced to amine by reductive amination using a reducing agent. ${ }^{13}$ Therefore, $\mathrm{NaBH}_{4}$ solution was added ( 2 equiv. with respect to glutaraldehyde) to crosslinked peptide hydrogels.

As soon as the reducing agent was added into the gel, the gel collapsed immediately. The reduced peptide gels showed no absorbance at $265 \mathrm{~nm}$ (Fig. 2A) and no $\beta$-sheet structure was observed (Fig. 2B). Reduction of imines to amines locked the system by the formation of covalent bonds instead of dynamic covalent bonds, therefore, the peptide self-assembly was disturbed and the peptides lost their $\beta$-sheet structure and the gel formation was prevented.

We further investigated the degree of deprotonated side chain amines by zeta potential analysis because only deprotonated amines can react with glutaraldehyde. Therefore, zeta potentials were measured at different $\mathrm{pH}$ values by titration of the peptide solution with $0.1 \mathrm{M} \mathrm{NaOH}$ (Fig. S7†). Despite the positive zeta potential $(\sim 15 \mathrm{mV})$, peptide molecules can form gel at around $\mathrm{pH}$ 7.5. The positive zeta potential indicates that amines are not fully neutralized (Fig. S7 $\dagger$ ).

The complete neutralization takes place when $\mathrm{pH}$ is above 8. Therefore, we investigated the gelation kinetics at minimum $\mathrm{pH}$ of gelation (7.5) and at pH 10 (Fig. S8 and S9†) to ensure the entire deprotonation of amines.

Gelation kinetics and viscoelastic properties are important material characteristics indicating the potential use of a hydrogel. Oscillatory rheology was used to monitor the mechanical stiffness and elasticity of the gel systems. Gelation kinetics was determined by a time-sweep test within the linear viscoelastic range. Within $75 \mathrm{~min}$, storage moduli, the energy stored during deformation, of all groups reached a plateau demon-
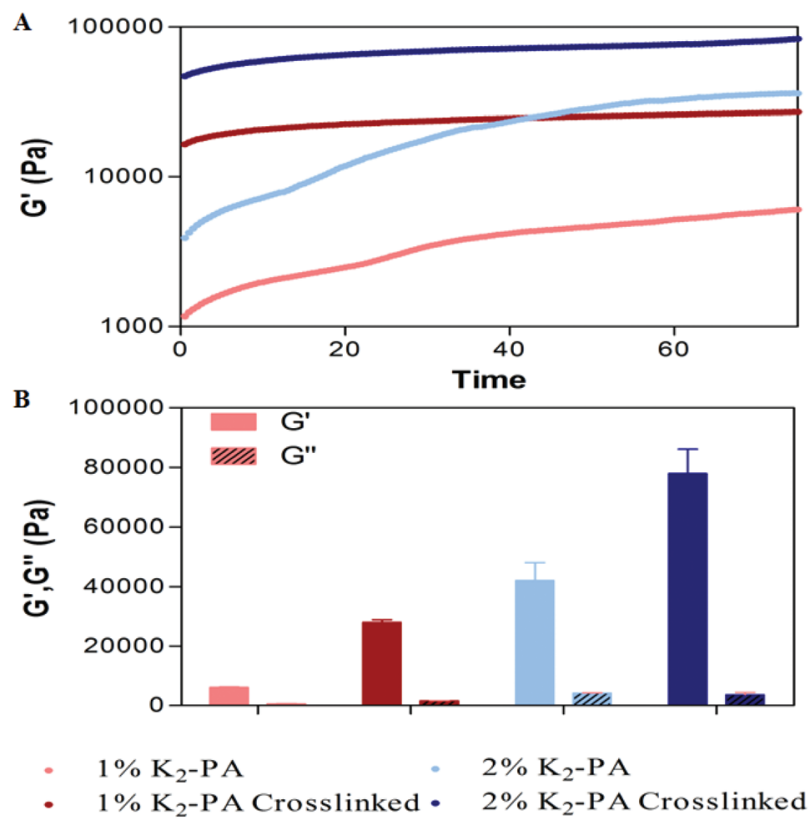

Fig. 3 Rheological characterization of the gels. (A) Gelation kinetics and $(\mathrm{B})$ equilibrium moduli at $\mathrm{pH} 7.5$. 
strating the complete gelation (Fig. 3A, S8a †). However, storage moduli of non-covalently self-assembled peptide gels showed a greater ramp at $\mathrm{pH} 7.5$ (Fig. 3A) when compared to $\mathrm{pH} 10$ (Fig. S8a $\dagger$ ), which reached a stationary phase at the earlier periods. This result indicated that the self-assembly process was completed at an increased rate under basic conditions. In addition, covalent crosslinking through glutaraldehyde addition resulted in rapid completion of the gelation process by locking the mobile PA molecules within the self-assembled system both at $\mathrm{pH} 7.5$ and $\mathrm{pH} 10$ (Fig. S8a $\dagger$ ) when compared to regular PA gel systems.

For all the groups, storage moduli $\left(G^{\prime}\right)$ were greater than loss moduli $\left(G^{\prime \prime}\right)$ confirming the gel character of the resulting

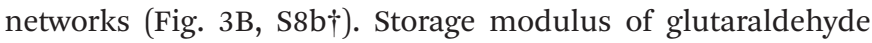
crosslinked samples was significantly higher for all groups when compared to their self-assembled PA controls showing that the crosslinking resulted in the formation of stiffer gels (Fig. 3B, S8b $\dagger$ ). Another important point to elucidate is the effect of crosslinking on the mechanical properties $\left(G^{\prime \prime} / G^{\prime}\right.$ ratio or loss (damping) factor). Gelation takes place when the $G^{\prime \prime} / G^{\prime}$ value is below 1 and a smaller damping factor refers to a more pronounced elastic character against a viscous character.

Storage moduli of glutaraldehyde crosslinked samples were significantly higher than non-covalently self-assembled PA controls while their loss moduli were comparable. Covalent crosslinking resulted in a smaller damping factor indicating the formation of stronger gels. Furthermore, the peptide formed stronger gels at $\mathrm{pH} 10$ (Fig. S10 $\dagger$ ) than at $\mathrm{pH} 7.5$ (Fig. S10 $\dagger$ ). The zeta potential analysis (Fig. S7†) at pH 7.5 indicated the presence of partially protonated amines. The positively charged amines cause repulsion among the peptide molecules and therefore weakening of the gel formation. In the case of glutaraldehyde crosslinking at $\mathrm{pH} 7.5$, the protonated amines lose their nucleophilicity and therefore cannot attack the aldehyde. We can conclude that the degree of crosslinking at $\mathrm{pH} 7.5$ is lesser than at $\mathrm{pH} 10$.

To investigate the viscoelastic properties of the resulting gels, we performed an amplitude sweep test showing the relationship between the storage modulus and strain amplitudes. Within the linear viscoelastic (LVE) range, gels maintained their storage moduli at constant values independent of increasing strain values. When a certain limit of LVE (limiting strain amplitude (LSA)) was exceeded, the gels showed a transition from linear to nonlinear viscoelastic behavior and the storage values started to decrease under increased strain values (Fig. 4A, S9a †). For all the groups, the limiting strain amplitude of glutaraldehyde crosslinked samples was approximately six times higher than that of self-assembled peptide nanofiber controls. This increase in LSA showed that compared to noncovalent networks, glutaraldehyde crosslinked PA gels can withstand six times higher shear strain. Covalent crosslinking supported the increased resistance to deformation and elasticity of the resulting networks.

To investigate the self-healing ability, in other words recovery after high shear loads, we performed a thixotrophic test
A
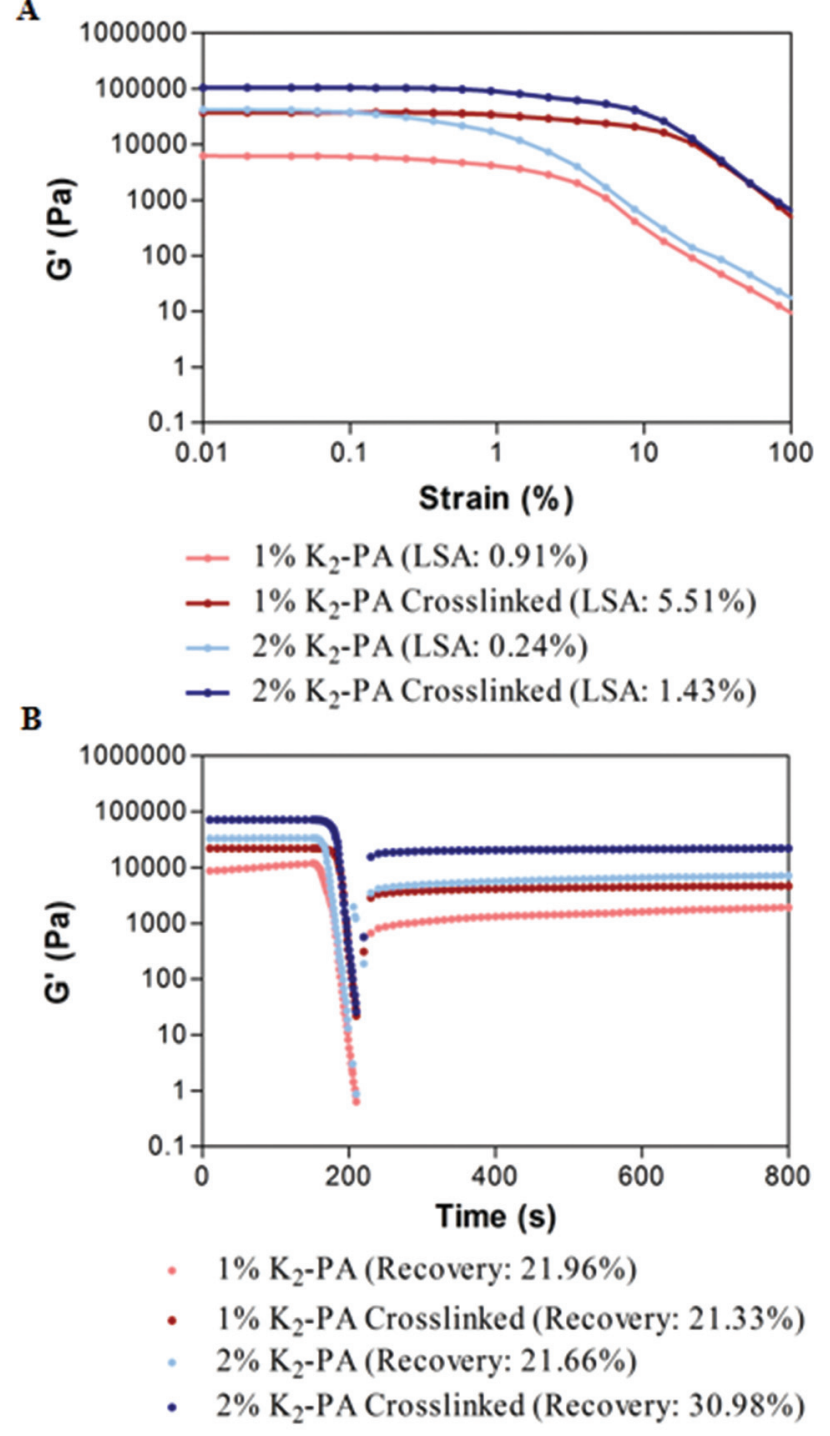

Fig. 4 (A) Amplitude sweep test and (B) Self-healing of the gels at $\mathrm{pH} 7.5$.

under increasing strain amplitudes far beyond the linear viscoelastic range up to $1000 \%$. At this range, all noncovalent interactions were expected to be broken to completely deform the network. When the shear load was removed, recovery of the noncovalent bonds was measured. The recovery of the glutaraldehyde crosslinked samples was comparable to their noncovalently self-assembled PA controls and no significant decrease was observed in the self-healing ability of the gels after covalent crosslinking (Fig. 4B, S9b $\dagger$ ). After deformation, noncovalent interactions driving the self-assembly of PA molecules were similarly restored both for noncovalently selfassembled PA gels and glutaraldehyde crosslinked PA gels. Therefore, these results indicate that the glutaraldehyde crosslinking increased the mechanical stability of PA gels, support the load bearing capacity and elasticity of the resulting networks, and also as an additional advantage, did not harm 


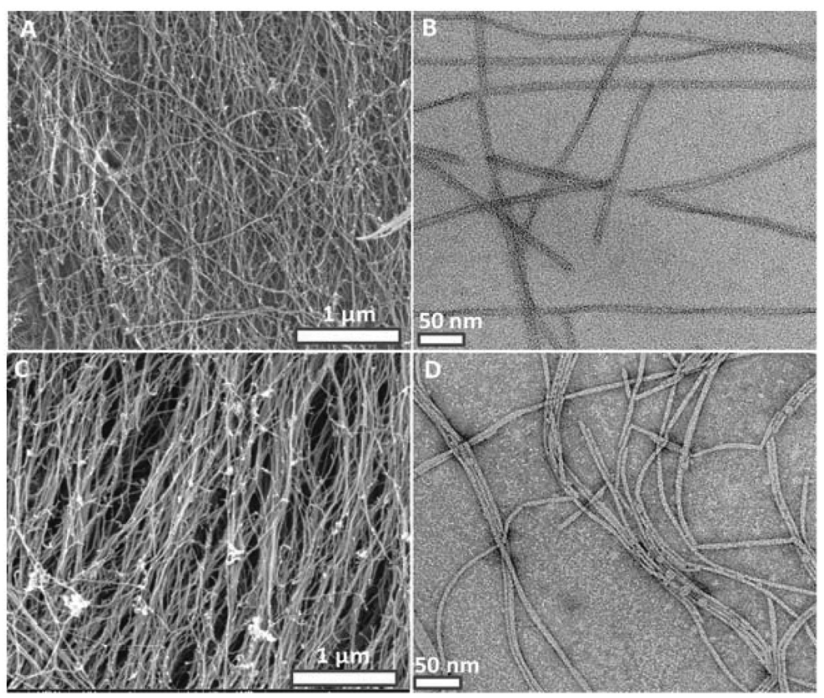

Fig. 5 SEM and TEM images of the peptide gels (A, B) and crosslinked peptide gels $(C, D)$ at $\mathrm{pH}$ 7.5.

the self-healing ability of noncovalent interactions within the self-assembled network.

Interestingly, the dynamic crosslinking process did not affect the nanofiber morphology, porosity and fibrous structure of the peptide gels (Fig. 5C, D and S4, S5, S6 $\dagger$ ). SEM (Fig. 5A, C) and TEM (Fig. 5B, D) images show no difference in width and length of the peptide nanofibers. Crosslinking at the molecular level is observed by UV spectroscopy (Fig. 2A) but we could not observe distinct morphological changes at the nanometer level. This could be due to dominant intrafibrillar crosslinking rather than interfibrillar crosslinking. UV-Vis, CD, SEM, TEM characterizations and self-healing ability of the crosslinked peptide gels prove the presence of a dynamic covalent imine bonding.

\section{Conclusion}

In this work, we have developed a novel supramolecular peptide nanostructure system, which could be crosslinked in a dynamic manner. This approach facilitates tuning and enhancing the viscoelastic characteristics of the self-assembled peptide gels. Besides improved mechanical stability, the peptide gels also conserved their porous, fibrous structures and self-healing characteristics. The viscoelastic properties of the peptide gels could further be tuned by addition of changing ratios of glutaraldehyde or addition of shorter or longer length dialdehydes.

\section{Notes and references}

1 H. Cui, M. J. Webber and S. I. Stupp, Self-assembly of peptide amphiphiles: from molecules to nanostructures to biomaterials, Biopolymers, 2010, 94(1), 1-18.
2 R. V. Ulijn and A. M. Smith, Designing peptide based nanomaterials, Chem. Soc. Rev., 2008, 37(4), 664-675.

3 (a) A. Dasgupta, J. H. Mondal and D. Das, Peptide hydrogels, RSC Adv., 2013, 3(24), 9117-9149; (b) H. Hosseinkhani, P. D. Hong and D. S. Yu, SelfAssembled Proteins and Peptides for Regenerative Medicine, Chem. Rev., 2013, 113(7), 4837-4861; (c) A. M. Jonker, D. W. P. M. Lowik and J. C. M. van Hest, Peptide- and Protein-Based Hydrogels, Chem. Mater., 2012, 24(5), 759773; (d) S. Bhattacharjee and S. Bhattacharya, Phthalate mediated hydrogelation of a pyrene based system: a novel scaffold for shape-persistent, self-healing luminescent soft material, J. Mater. Chem. A, 2014, 2(42), 17889-17898; (e) P. Moitra, K. Kumar, P. Kondaiah and S. Bhattacharya, Efficacious Anticancer Drug Delivery Mediated by a pH-Sensitive Self-Assembly of a Conserved Tripeptide Derived from Tyrosine Kinase NGF Receptor, Angew. Chem., Int. Ed., 2014, 53(4), 1113-1117.

4 X. Zhao, F. Pan, H. Xu, M. Yaseen, H. Shan, C. A. Hauser, S. Zhang and J. R. Lu, Molecular self-assembly and applications of designer peptide amphiphiles, Chem. Soc. Rev., 2010, 39(9), 3480-3498.

5 M. A. Khalily, O. Ustahuseyin, R. Garifullin, R. Genc and M. O. Guler, A supramolecular peptide nanofiber templated Pd nanocatalyst for efficient Suzuki coupling reactions under aqueous conditions, Chem. Commun., 2012, 48(92), 11358-11360.

6 M. O. Guler and S. I. Stupp, A self-assembled nanofiber catalyst for ester hydrolysis, J. Am. Chem. Soc., 2007, 129(40), 12082-12083.

7 S. K. M. Nalluri, C. Berdugo, N. Javid, P. W. J. M. Frederix and R. V. Ulijn, Biocatalytic Self-Assembly of Supramolecular Charge-Transfer Nanostructures Based on n-Type Semiconductor-Appended Peptides, Angew. Chem., Int. Ed., 2014, 53(23), 5882-5887.

8 Y. Ding, Y. Li, M. Qin, Y. Cao and W. Wang, Photo-CrossLinking Approach to Engineering Small Tyrosine-Containing Peptide Hydrogels with Enhanced Mechanical Stability, Langmuir, 2013, 29(43), 13299-13306.

9 J. C. Stendahl, M. S. Rao, M. O. Guler and S. I. Stupp, Intermolecular forces in the self-assembly of peptide amphiphile nanofibers, Adv. Funct. Mater., 2006, 16(4), 499-508.

10 W. Y. Seow and C. A. E. Hauser, Tunable Mechanical Properties of Ultrasmall Peptide Hydrogels by Crosslinking and Functionalization to Achieve the 3D Distribution of Cells, Adv. Healthcare Mater., 2013, 2(9), 1219-1223.

11 J. Boekhoven, J. M. Poolman, C. Maity, F. Li, L. van der Mee, C. B. Minkenberg, E. Mendes, J. H. van Esch and R. Eelkema, Catalytic control over supramolecular gel formation, Nat. Chem., 2013, 5(5), 433-437.

12 Y. Li, Y. Ding, M. Qin, Y. Cao and W. Wang, An enzymeassisted nanoparticle crosslinking approach to enhance the mechanical strength of peptide-based supramolecular hydrogels, Chem. Commun., 2013, 49(77), 8653-8655.

13 M. E. Belowich and J. F. Stoddart, Dynamic imine chemistry, Chem. Soc. Rev., 2012, 41(6), 2003-2024. 
14 (a) L. H. H. O. Damink, P. J. Dijkstra, M. J. A. Vanluyn, P. B. Vanwachem, P. Nieuwenhuis and J. Feijen, Glutaraldehyde as a Cross-Linking Agent for Collagen-Based Biomaterials, J. Mater. Sci. Mater. Med., 1995, 6(8), 460-472; (b) I. Migneault, C. Dartiguenave, M. J. Bertrand and K. C. Waldron, Glutaraldehyde: behavior in aqueous solution, reaction with proteins, and application to enzyme crosslinking, Biotechniques, 2004, 37(5), 790796.

15 J. H. Bowes and C. W. Cater, The interaction of aldehydes with collagen, Biochim. Biophys. Acta, 1968, 168(2), 341352 . 